distinguishes it from the other $\mathrm{B}$ vitamins hitherto described. Miss Roscoe has found no relationship between fat in the diet and the need for vitamin $B_{2}$ : fat lessens the need for vitamin $B_{1}$.

Dr. W. R. Aykroyd has found that the endosperms of wheat, rice, and maize contain little vitamin $B_{1}$ as compared with the embyro or whole grain. Parboiled rice, however, even after milling, contains definite amounts, due apparently to diffusion inwards from the germ and pericarp during the boiling. Maize has been found a good source of vitamin $\mathrm{B}_{2}$, as good as wheat, and better than rice. Pellagra, however, occurs among maize-eating peoples and is rarely observed where rice is the staple food. This result is against the theory that pellagra is due to a simple deficiency of vitamin $\mathrm{B}_{2}$

Miss E. M. Hume and Miss H. H. Smith have compared the effect on bone calcification in rats of diets low and rich in ergosterol, the animals being given a complete synthetic diet without vitamin $\mathrm{D}$ but irradiated daily. The bone ash was slightly lower in the animals on the low ergosterol diet which were not irradiated than in any of the other groups. The diet used contained no fat : many of the rats developed scaliness of the tails, which has been attributed to absence of fat from the diet. Hume and Smith observed the condition, however, in other rats on a diet containing fat, so that it must be due to some other defect in the diet.

Dr. N. S. Lucas has reinvestigated the permeability of human epidermis to radiations between $4370 \mathrm{~A}$. and $2400 \mathrm{~A}$. The transparency has been much underestimated, since considerable scattering of the incident light occurs, so that it fails to reach the slit of the spectrograph, although it may ultimately be transmitted by the skin at a distance from its point of entry. When allowance is made for this, by producing scattering of the light before it reaches the skin, it is found that 55 per cent is transmitted at $3130 \mathrm{~A}$. and 26 per cent at $2940 \mathrm{~A}$. The absorption curve agrees closely with those of casein, serum albumin, tryptophane, and tyrosine.

Dr. Smedley-MacLean and Miss Pearce have shown that when a large excess of peroxide acts on oleic acid at $95^{\circ} \mathrm{C}$., less than 20 per cent of the carbon appears as formic, carbonic, and acetic acids, acids with 15 and 12 carbon atoms being obtained, that is, oxidation occurs first at the double bond followed by oxidation of the 18 carbon chain in the $\gamma$ position. In the presence of a cupric salt, the oxidation proceeds much further, 70 per cent appearing as the lower acids. At $60^{\circ} \mathrm{C}$. the chief products are 4-keto 7 -hydroxy caprylic and succinic acids with 20 per cent of the lower acids. The evidence lends no support to the view that fatty acids are broken down by $\beta$-oxidation in the body. The presence of succinic acid is of interest since an oxidising enzyme for this acid is widely distributed throughout the tissues.

Among other researches with which the Report deals may be mentioned those of Dr. Robison on calcification in vitro and on the chemistry of the hexosephosphates and the work of Dr. V. Korenchevsky on the amount of fat in castrated and cryptorchid animals: degeneration or removal of the seminiferous tissue leads to an increase in fat deposition.

\title{
A New Illumination Device for Microscopy.
}

$\mathrm{T}^{\mathrm{H}}$ HE microscopic examination of opaque materials and the surface structure of natural objects have recently attracted much attention. The root idea of the Lieberkühn has been embodied in various types of ring illuminator in which the light may reach an annular reflector from below, or may be dealt with in a manner more analogous to the vertical illuminator.

Messrs. Ernst Leitz have recently brought out improved apparatus of the latter kind in which the light reaches the object from an annular illuminator around the objective, but avoiding the restriction in the size of the object which has been necessary in some arrangements. A small 8-volt lamp and condenser project a roughly parallel beam which is reflected downwards by an annular mirror held at $45^{\circ}$ to the beam and to the axis of the microscope tube. The reflector is pierced with a central aperture which transmits the upward image-forming rays from the objective. The annular beam thus produced from the reflector enters an annular lens (or reflector system for the higher powers) and is focused on the object without travers. ing the objective itself. Thus the disadvantages of back reflections and stray light are avoided. The general arrangement of the parts is shown in the accompanying illustration (Fig. 1).

A special series of fifteen new objectives, comprising 'dry ", 'water immersion ', and 'oil immersion ' lenses has been produced, in which the low-power lenses are designed to work without a cover glass over the object. Each of the low-power lenses has its own ring condenser, and rapid transition from one object to another is facilitated by the use of a convenient objective changer which clamps firmly and definitely into position. The lamp and condenser system need not be changed.

One advantage of apparatus of this kind is that the illumination can be controlled with much greater precision than with any more or less haphazard reflector arrangement, and that a systematic study of objects can therefore be commenced with less uncertainty. The interpretation of the images in some cases, especially with the higher powers, is not an easy matter,

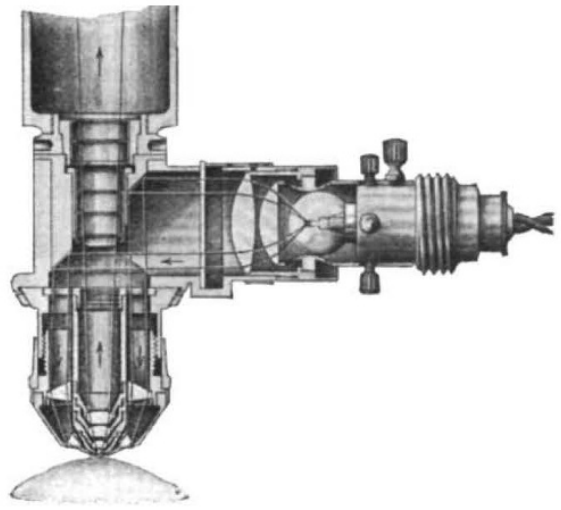

Fia. 1.

and will require careful study; diffraction effects can easily be mistaken for surface colour; neverthe. less, it is clear that new investigations are possible. The well-illustrated catalogue of this 'Ultropaque Illuminator' gives photographs of various objects, amongst which are vegetable tissues, skin, chromatophores, leather, wood-sections, fabrics, and so on. This method may also have important possibilities in metallography.

L. C. M.

No. 3241, VoL. 128] 